\title{
Prevalence of major infections and adverse outcomes among hospitalized. ST-elevation myocardial infarction patients in Florida, 2006
}

\author{
Michelle C Nash ${ }^{1 *}$, Joel A Strom² and Elizabeth B Pathak
}

\begin{abstract}
Background: ST-elevation myocardial infarction (STEMI) patients have risk factors and co-morbidities and require procedures predisposing to healthcare acquired infections (HAls). As few data exist on the extent and consequences of infections among these patients, the prevalence, predictors, and potential complications of major infections among hospitalized STEMI patients at all Florida acute care hospitals during 2006 were analyzed.

Methods: Sociodemographic characteristics, risk factors, co-morbidities, procedures, complications, and mortality were analyzed from hospital discharge data for 11, 879 STEMI patients age $\geq 18$ years. We used multivariable logistic regression modeling to examine and adjust for multiple potential predictors of any infection, bloodstream infection (BSI), pneumonia, surgical site infection (SSI), and urinary tract infection (UTI).

Results: There were 2, 562 infections among $16.6 \%$ of STEMI patients; $6.2 \%$ of patients had $\geq 2$ infections. The most prevalent HAls were UTIs (6.0\%), pneumonia (4.6\%), SSIs (4.1\%), and BSIs (2.6\%). Women were at 29\% greater risk, Blacks had 23\% greater risk, and HAl risk increased 11\% with each 5 year increase in age. PCI was the only protective major procedure (OR 0.81, 95\% Cl, 0.69-0.95, $\mathrm{p}<.05$ ). HAl lengthened hospital stays. STEMI patients with a BSI were almost 5 times more likely ( $31.3 \%$ vs. $6.5 \%, p<.0001)$, and those with pneumonia were 3 times more likely $(19.6 \%$ vs. $6.5 \%, p<.0001)$ to die before discharge.
\end{abstract}

Conclusions: The protective effect of $\mathrm{PCl}$ on risk of infection is likely mediated by its many benefits, including reduced length of hospitalizations.

\section{Background}

Approximately two million health care acquired infections (HAIs) occur annually (affecting $5-10 \%$ of all inpatients and $25 \%$ of intensive care unit (ICU) patients), contributing to an estimated 90,000 deaths and $\$ 4.5$ to $\$ 5.7$ billion dollars in excess healthcare expenditures $[1,2]$. HAI patients average five times the length of stay (LOS) and treatment cost and six times the in-hospital mortality versus those without HAIs [3]. Over $80 \%$ of HAIs belong to four categories: urinary tract infection (UTI) (35\% of all infections), surgical site infection (SSI) (20\%), bloodstream infection (BSI) (15\%), and pneumonia (15\%) [2]. BSIs and pneumonia most significantly

\footnotetext{
* Correspondence: mnash@health.usf.edu

'Department of Epidemiology and Biostatistics, College of Public Health, University of South Florida, (13201 Bruce B. Downs Blvd.), Tampa, (33612), USA

Full list of author information is available at the end of the article
}

increase mortality [2]. UTIs, despite their prevalence, result in the lowest additional treatment costs and excess mortality. Risks of hospital acquired UTI include: female sex, non-white ethnicity, co-morbidities, unit of admission, indwelling catheters, invasive procedures, and mechanical ventilation [4]. Mechanical ventilator use is also strongly associated with development of pneumonia [2], while indwelling intravascular catheters, implanted medical devices, LOS in ICU or surgical ward, and invasive procedures are associated with bacteremias [5].

Few studies have examined predictors of HAIs among acute myocardial infarction (AMI) patients, especially ST-elevation myocardial infarction (STEMI) [6]. Among cardiac care unit (CCU) occupants, pneumonia, BSIs, and UTIs were strongly associated with invasive device implantation (i.e. mechanical ventilator, catheter). However, overall incidence of CCU-acquired HAIs was less than in other ICUs, attributable to less frequent use of 
invasive devices [7]. Reperfusion therapy may cause HAI, by frequent use of invasive devices and procedures and procedure-related complications, e.g. contrastinduced nephropathy. While infrequently utilized for STEMI patients, surgical revascularization increases the risk for surgical site infections, pneumonia, and UTIs $[8,9]$.

To define the prevalence and clinical impact of HAI in STEMI patients, we examined the prevalence, predictors, and potential complications of major infections among hospitalized STEMI patients at all acute care hospitals in Florida during 2006 (before the October 2007 Medicare mandate to report "present on admission" data for infections), thereby providing baseline population-based and unselected prevalence data on infections in a highrisk patient population.

\section{Methods}

\section{Study Population and Data Sources}

All adults age $\geq 18$ years with a primary diagnosis of STEMI admitted to non-Veterans Administration acute care hospitals in 2006 were included. Surveillance data was obtained from the Florida Agency for Health Care Administration Hospital Discharge Database, which captures $100 \%$ of hospital discharges. Patient variables analyzed: age, race/ethnicity, gender, insurance type, type and source of admission, admitting diagnosis, principal diagnosis, secondary diagnoses (up to 30 per patient), procedures (up to 30 per patient), length of stay, and vital status at discharge. Diagnoses and procedures were coded using the International Classification of Diseases ( $9^{\text {th }}$ Revision), Clinical Modification (ICD-9-CM). The University of South Florida IRB granted the study exempt status.

A total of 14, 148 patient admissions carried a principal diagnosis of STEMI in 2006, identified by the principal diagnosis ICD-9-CM codes 410.0 to 410.6 and 410.8, with a fifth digit of 0 or 1 . After excluding transfers ( $\mathrm{n}$ $=2,269$ ), because we could not link separate hospital records, 11, 879 STEMI patients remained.

\section{Variable Definitions}

Infections were identified by secondary diagnosis ICD-9$\mathrm{CM}$ codes representing conditions listed in CDC/NHSN surveillance definition of health care-associated infection and criteria for specific types of infections in the acute care setting (2008) [10]. We added to a list of HAI ICD9-CM codes compiled by Sherman et al. (2006) [11] by completing our own review of ICD-9-CM infection codes. Patient risk factors, co-morbidities, and procedures were identified from the thirty ICD-9-CM procedure and secondary diagnosis codes.

Based on CDC surveillance guidelines, we grouped infection codes into the following categories: (1) BSI, (2) pneumonia, (3) SSI, (4) UTI, (5) central nervous system illness, (6) gastrointestinal illness, (7) lower respiratory tract infection, (8) skin and soft tissue infection, (9) bone and joint infection, (10) cardiovascular infection, and (11) ear, eye, nose, and throat infection. Only categories 1-4 included enough patients to warrant separate modeling of predictors. Categories 6-11 were collapsed into an "other infection" group (there were no cases in category 5), and included patients with secondary diagnosis ICD-9-CM codes for site unspecified infections. Patients with $\geq 2$ infections could be included in more than one group.

Patient characteristics analyzed were age, sex, race/ ethnicity, and socioeconomic status. Asians/Pacific Islanders, American Indians/Alaskan Natives were grouped with whites due to their very small numbers. Insurance type (Medicaid, other state/government, self pay, underinsured, and charity) was a proxy for low income. Risk factors analyzed were cigarette smoking, alcohol, and drug abuse. Our analysis included six serious co-morbidities (chronic bronchitis, COPD, diabetes mellitus, chronic kidney diseases, cardiomyopathy, and valve disorders), and the following procedures: cardiac catheterization, percutaneous coronary intervention (PCI), cardiac surgical procedures, indwelling arterial or venous catheter, pacemakers and/or implantable defibrillators, dialysis, and blood transfusion. Hospital LOS was the total number of days between admission and discharge. ICU or CCU stay was a dichotomous variable (yes/no) based on the presence or absence of ICU/CCU financial charges in the discharge record.

\section{Statistical Analyses}

We evaluated the proportion of STEMI patients with any infection and for each category, along with the total number of infections present and the prevalence of multiple infections. We used multivariable logistic regression modeling to examine and adjust for multiple potential predictors of any infection, BSI, pneumonia, SSI, and UTI. The 9, 910 patients with no diagnosed infection comprised the comparison group for all models.

Several important co-morbidities (acute renal failure, heart failure), procedures (mechanical ventilation, SwanGanz catheter), and outcomes (admission to the ICU/ CCU, hospital LOS), important potential complications of infection, were not included in the predictive models because the temporal relationship to the onset of infection could not be determined, e.g. whether ICU stay resulted in or was a consequence of an infection. The prevalence of each potential infectious complication was compared to the prevalence among non-infected patients using a chi-square test of homogeneity. The inhospital mortality for each infection group was 
compared to non-infected STEMI patients. We used SAS version 9.1.3 (SAS Institute, Cary, North Carolina) for all analyses.

\section{Results}

There were 2, 562 infections among 1, 969 (16.6\%) STEMI patients; 739 (6.2\%) patients had $\geq 2$ infections. UTIs (6.0\%) were most prevalent, followed by pneumonia (4.6\%), SSIs (4.1\%), and BSIs (2.6\%). Their relative frequencies were $36.1 \%, 27.7 \%, 24.7 \%$, and $15.7 \%$, respectively. Lower respiratory tract, cardiovascular, bone and joint, eye, ear, nose, and throat, gastrointestinal, skin and soft tissue, and non-specific bacterial infections (other infection) comprised $4.2 \%$ of patients (Table 1). Thirty-seven $(0.3 \%)$ patients had an intra-cardiac or pericardial infection. Overall, STEMI patients were older, more likely to be white and male, and a nearly one-third smoked (28.9\%) and had diabetes (27.1\%). The most common procedure was cardiac catheterization (66.9\%), followed by PCI $(59.0 \%)$. Infected patients were older, less likely to be smokers and receive PCI, and had more co-morbidities than patients with no infection (Table 2). The majority of infected STEMI patients underwent at least one minor or major procedure.

After multivariable adjustment, all other risk factors and co-morbidities examined were associated with increased risk of infection, except for cigarette smoking (OR 0.76, 95\% CI 0.66-0.87, p < .01) (Table 3). Women

Table 1 Infections among hospitalized STEMI patients ( $n=11,879$ )* in Florida, 2006.

\begin{tabular}{|c|c|}
\hline Infection Categories $^{\dagger}$ & Frequency of Infection ${ }^{\ddagger} \%$ (n) \\
\hline Infected STEMI Patients & $16.6(1,969)$ \\
\hline Blood Stream Infection (BSI) & $2.6(310)$ \\
\hline Any additional infection & $75.0(233)$ \\
\hline No additional infection & $25.0(77)$ \\
\hline Pneumonia & $4.6(550)$ \\
\hline Any additional infection & $41.0(224)$ \\
\hline No additional infection & $59.0(326)$ \\
\hline Surgical Site Infection (SSI) & $4.1(491)$ \\
\hline Any additional infection & $30.0(145)$ \\
\hline No additional infection & $70.0(346)$ \\
\hline Urinary Tract Infection (UTI) & $6.0(708)$ \\
\hline Any additional infection & $35.0(248)$ \\
\hline No additional infection & $65.0(460)$ \\
\hline All Other Infections ${ }^{\S}$ & $4.2(503)$ \\
\hline
\end{tabular}

*Excludes patients who were transferred to another hospital. tPatients can appear in more than one infection category. ¥There were a total of 2, 562 infections.

SIncludes lower respiratory, gastrointestinal, cardiovascular, bone and joint, and ear, eye, nose \& throat, skin and soft tissue, and site unspecified bacterial infections. and Blacks had a $29 \%$ and $23 \%$ greater risk, respectively, while the risk of infection increased 11\% with each 5 year increase in age. Most procedures conveyed an increased risk - the notable exception was the lower risk associated with PCI (OR 0.81, 95\% CI, 0.69-0.95, $\mathrm{p}<.01$ ).

\section{Bloodstream Infections}

BSIs were the most serious but least common type of HAI (Table 3). Valve disease (OR 2.22, 95\% CI 1.263.92, $\mathrm{p}<.01$ ) and COPD (OR 1.80, 95\% CI 1.03-3.14, p $<.05)$ conveyed particular risk. A trend toward elevated prevalence was observed among Hispanics, older patients, and those with chronic kidney diseases (including ESRD) ( $\mathrm{p}=\mathrm{n} . \mathrm{s}$.). Procedures with the strongest association were an indwelling arterial or venous catheter (OR 6.99, 95\% CI 3.93-12.41, $\mathrm{p}<.0001)$, dialysis (OR 6.97, 95\% CI 2.81-17.26, $\mathrm{p}<.0001)$, and blood transfusion (OR 2.10, 95\% 1.07-4.11, p <.05). BSI sufferers were more likely than non-infected patients to develop acute renal failure $(51.3 \%$ vs. $5.3 \%, \mathrm{p}<.0001)$ or heart failure $(58.8 \%$ vs. $18.0 \%, \mathrm{p}<.0001)$, and were almost 5 times as likely to die before discharge $(31.3 \%$ vs. $6.5 \%, \mathrm{p}$ $<$.0001) (Table 4).

\section{Pneumonia}

Pneumonia risk increased with alcohol abuse (OR 2.31, 95\% CI 1.33-4.01, $\mathrm{p}<.01$ ), chronic bronchitis (OR 4.30, 95\% CI 2.82-6.56, $\mathrm{p}<.0001$ ), and COPD (OR 2.28, 95\% CI 1.75-2.96, $\mathrm{p}<.0001)$, but not with sociodemographic characteristics (Table 3). Indwelling intravascular catheters (OR 3.93, 95\% CI 2.79-5.55, $\mathrm{p}<.0001)$, dialysis (OR $3.55,95 \%$ CI 1.76-7.09, $\mathrm{p}<.01)$, and cardiac surgical procedures (OR 1.54, 95\% CI 1.02-2.33, $\mathrm{p}<.05$ ) were associated with an increased risk. Although mechanical ventilation has been reported to convey a strong risk for development of pneumonia, [12] only $13.1 \%$ of the STEMI patients with pneumonia were ventilated during their hospital stay (Table 4). Pneumonia was complicated more frequently by acute renal failure $(30.6 \%$ vs. $5.3 \%, \mathrm{p}<.0001)$, heart failure $(63.5 \%$ vs. $18.0 \%, \mathrm{p}<$ $.0001)$, and patients were 3 times as likely to in-hospital $(19.6 \%$ vs. $6.5 \%, \mathrm{p}<.0001)$.

\section{Surgical Site Infections}

Cardiac catheterization (OR 2.89, 95\% CI 1.98-4.22, p < .0001) was the strongest predictor of SSI, followed by dialysis (OR 2.61, 95\% CI 1.10-6.17, $\mathrm{p}<.05$ ), indwelling intravascular catheters (OR 1.98, 95\% CI 1.30-3.03, p < .05 ), and blood transfusion (OR 1.62, 95\% CI 1.08-2.45, $\mathrm{p}<.05)$. However, PCI was associated with a lower risk of SSI (OR 0.63, 95\% CI 0.46-0.88, p <.01). Except for the lower risk of infection associated with cigarette smoking (OR 0.70, 95\% CI 0.53-0.91, p <.01), co-morbidities and risk factors did not impact risk of SSI. We 
Table 2 Characteristics of hospitalized STEMI patients $(n=11,879) *$ by infection status, Florida 2006.

\begin{tabular}{|c|c|c|c|c|c|c|c|}
\hline & $\begin{array}{c}\text { All STEMI } \\
\text { Patients } \\
\mathrm{n}=11,879 \\
\%(n) \\
\end{array}$ & $\begin{array}{c}\text { Non-Infected } \\
\text { STEMI Patients } \\
\mathrm{n}=9,910 \\
\%(n) \\
\end{array}$ & $\begin{array}{c}\text { Blood Stream } \\
\text { Infection }^{+} \\
\mathrm{n}=310 \\
\%(n)\end{array}$ & $\begin{array}{l}\text { Pneumonia } \\
\mathrm{n}=550 \\
\%(n)\end{array}$ & $\begin{array}{c}\text { Surgical Site } \\
\text { Infection } \\
n=491 \\
\%(n)\end{array}$ & $\begin{array}{l}\text { Urinary Tract } \\
\text { Infection } \\
\mathrm{n}=708 \\
\%(n)\end{array}$ & $\begin{array}{c}\text { All Other } \\
\text { Infections }^{\ddagger} \\
\mathrm{n}=503 \\
\% \text { (n) }\end{array}$ \\
\hline \multicolumn{8}{|l|}{$\begin{array}{l}\text { Patient } \\
\text { Sociodemographic } \\
\text { Characteristics } \wedge\end{array}$} \\
\hline \multicolumn{8}{|l|}{ Age } \\
\hline Age 18-34 Years & $0.9(107)$ & $1.0(100)$ & No cases** & No cases** & 0.6 (3) 㧊 & $0.3(2)^{* *}$ & $0.8(4)^{* *}$ \\
\hline Age 35-44 Years & $6.2(735)$ & $6.6(658)$ & $4.9(15)$ & $2.4(13)$ & $5.9(29)$ & $1.4(10)$ & $6.4(32)$ \\
\hline Age 45-54 Years & $18.7(3057)$ & 20.0 (1984) & $10.1(31)$ & $3.6(47)$ & $20.6(101)$ & $5.4(38)$ & $14.5(73)$ \\
\hline Age 55-64 Years & 23.9 (2837) & $24.9(2471)$ & $19.5(60)$ & $19.6(108)$ & $22.8(112)$ & $14.0(99)$ & 19.7 (99) \\
\hline Age 65-74 Years & $21.7(2573)$ & 21.4 (2116) & $24.7(76)$ & 24.9 (137) & $25.3(124)$ & $21.2(150)$ & $22.3(112)$ \\
\hline Age 75-84 Years & 18.5 (2196) & $17.0(1680)$ & $26.3(81)$ & $28.0(154)$ & $19.1(94)$ & $34.0(241)$ & $23.7(119)$ \\
\hline Age $85+$ Years & $10.2(1216)$ & $9.1(901)$ & $14.6(45)$ & $16.6(91)$ & $5.7(28)$ & 23.7 (168) & $12.7(64)$ \\
\hline \multicolumn{8}{|l|}{ Sex } \\
\hline Female & $33.7(4001)$ & $31.8(3149)$ & 38.6 (119) †† & $\begin{array}{c}38.6(212) \\
+\dagger\end{array}$ & $30.4(149)$ & $59.5(421)^{* *}$ & $42.9(216)^{* *}$ \\
\hline Male & 66.3 (7878) & $68.2(6761)$ & $61.4(189)$ & $61.5(338)$ & 69.7 (342) & $40.5(287)$ & $57.1(287)$ \\
\hline \multicolumn{8}{|l|}{ Race/Ethnicity } \\
\hline White & 83.1 (9868) & 83.3 (8256) & $78.3(241)$ & $81.8(450)$ & $81.5(400)$ & $82.2(582)$ & $82.3(414)$ \\
\hline Black & 6.7 (798) & $6.5(640)$ & $9.1(28)$ & $7.6(52)$ & $9.2(45)$ & $8.2(58)$ & $7.4(37)$ \\
\hline Hispanic & $10.2(1213)$ & $10.2(1014)$ & $12.7(39)$ & $10.6(58)$ & $9.4(46)$ & $9.6(68)$ & $10.3(52)$ \\
\hline \multicolumn{8}{|l|}{ Income by Proxy } \\
\hline $\begin{array}{l}\text { Low income (Medicaid, } \\
\text { self-pay, } \\
\text { uninsured) }\end{array}$ & 16.7 (1979) & $17.3(1711)$ & 13.0 (40) 㧊 & $10.6(58)^{* *}$ & $19.6(96)$ & $9.5(67)^{* *}$ & $15.9(80)$ \\
\hline All other insurance ${ }^{\S}$ & $83.3(9900)$ & $82.7(8199)$ & $87.0(268)$ & $89.5(492)$ & $80.5(395)$ & $90.5(641)$ & $84.1(423)$ \\
\hline \multicolumn{8}{|l|}{ Patient Risk Factors } \\
\hline Cigarette smoking & $28.9(3430)$ & $30.6(3030)$ & $14.9(46)^{* *}$ & $22.4(123)^{* *}$ & $24.4(120)+\dagger$ & $12.0(85)^{* *}$ & $23.7(119)+\dagger$ \\
\hline Alcohol abuse & $3.0(352)$ & $2.8(273)$ & $4.6(14)$ & 5.3 (29) t† & 4.9 (24) t† & $2.0(14)$ & $5.8(29)^{* *}$ \\
\hline Drug abuse & $2.4(287)$ & $2.4(237)$ & $2.3(7)$ & $2.2(12)$ & $3.3(16)$ & $1.6(11)$ & $4.2(21)$ 执 \\
\hline \multicolumn{8}{|l|}{ Patient Co-Morbidities } \\
\hline Chronic bronchitis & $2.7(317)$ & $2.2(213)$ & $6.8(21)^{* *}$ & $10.0(55)^{* *}$ & $4.3(21)$ t† & $5.1(36)^{* *}$ & $4.0(20)$ t† \\
\hline $\begin{array}{l}\text { Chronic obstructive } \\
\text { pulmonary } \\
\text { disease (COPD) }\end{array}$ & $16.2(1921)$ & $14.0(1382)$ & $28.3(87)^{* *}$ & $30.7(169)^{* *}$ & $20.8(102)^{* *}$ & $24.0(170)^{* *}$ & $37.0(186)^{* *}$ \\
\hline Diabetes & $27.1(3222)$ & $25.5(2527)$ & $35.1(108) \S \S$ & $34.2(188)^{* *}$ & 32.4 (159) †† & $38.6(273)^{* *}$ & $37.2(187)^{* *}$ \\
\hline Chronic kidney diseases & $9.6(1145)$ & $7.7(764)$ & $29.9(92)^{* *}$ & $22.7(125)^{* *}$ & $13.4(66)^{* *}$ & $23.2(164)^{* *}$ & $17.1(86)^{* *}$ \\
\hline Cardiomyopathy & $6.3(755)$ & $5.5(546)$ & $16.9(52)^{* *}$ & $12.7(70)^{* *}$ & $9.8(48)^{* *}$ & $11.0(78)^{* *}$ & $11.9(60)^{* *}$ \\
\hline Valve disorders & $10.1(1203)$ & $9.1(902)$ & $21.4(66)^{* *}$ & $16.0(88)^{* *}$ & $11.8(58)$ 㧊 & $16.2(115)^{* *}$ & $17.9(90)^{* *}$ \\
\hline \multicolumn{8}{|l|}{ Patient Procedures } \\
\hline Cardiac catheterization & $66.9(7941)$ & $67.7(6710)$ & $59.4(184)$ t† & $57.1(314)^{* *}$ & $80.7(396)^{* *}$ & $54.2(384)^{* *}$ & $65.4(329)$ \\
\hline $\begin{array}{l}\text { Percutaneous coronary } \\
\text { intervention } \\
\text { (PCI) }\end{array}$ & $59.0(7009)$ & $61.2(6065)$ & $41.6(128)^{* *}$ & $44.0(242)^{* *}$ & $62.1(305)$ & $41.0(290)^{* *}$ & $47.9(241)^{* *}$ \\
\hline $\begin{array}{l}\text { Cardiac surgical } \\
\text { procedures }\end{array}$ & $9.8(1168)$ & $8.4(830)$ & $24.4(75)^{* *}$ & $18.6(102)^{* *}$ & $19.4(95)^{* *}$ & $16.4(116)^{* *}$ & $24.1(121)^{* *}$ \\
\hline $\begin{array}{l}\text { Indwelling arterial or } \\
\text { venous } \\
\text { catheter }\end{array}$ & $6.0(707)$ & $3.6(360)$ & $45.2(140)^{* *}$ & $26.6(146)^{* *}$ & $18.1(89)^{* *}$ & $18.9(134)^{* *}$ & $20.9(105)^{* *}$ \\
\hline Pacemaker $^{\#}$ & $2.3(275)$ & $2.1(210)$ & $3.6(11)$ & $4.2(23)+\dagger$ & $4.9(24)^{* *}$ & $2.3(16)$ & $3.6(18) \neq \neq$ \\
\hline Dialysis & $1.5(172)$ & $0.7(68)$ & $16.5(51)^{* *}$ & $6.9(38)^{* *}$ & $6.5(32)^{* *}$ & $5.1(36)^{* *}$ & $6.6(33)^{* *}$ \\
\hline Blood transfusion & $6.7(800)$ & $5.1(507)$ & $26.6(82)^{* *}$ & $15.3(84)^{* *}$ & $15.3(75)^{* *}$ & $17.5(124)^{* *}$ & $15.5(78)^{* *}$ \\
\hline
\end{tabular}


Table 2 Characteristics of hospitalized STEMI patients $(n=11,879) *$ by infection status, Florida 2006. (Continued)

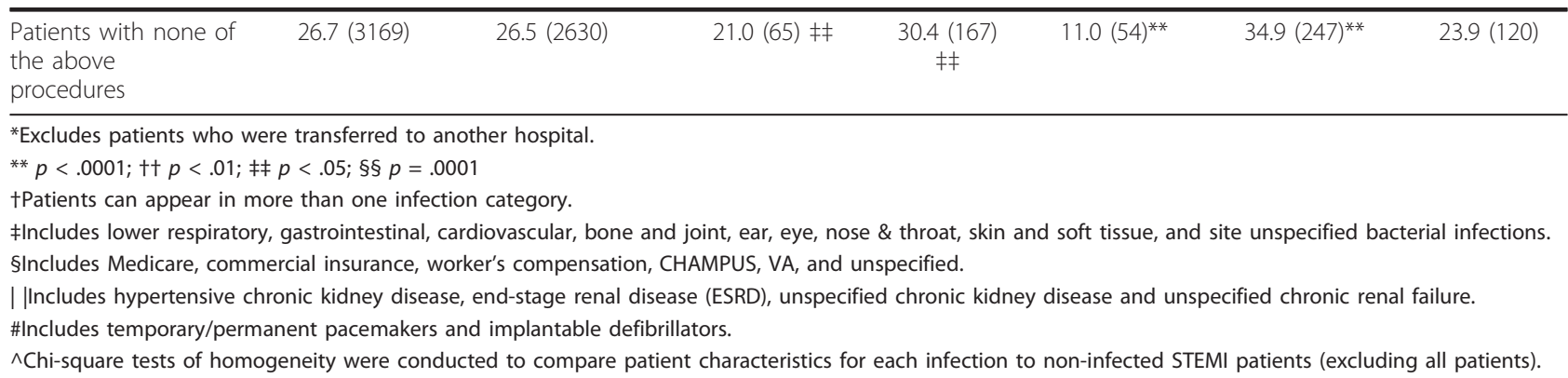

observed a moderate trend ( $\mathrm{p}=$ n.s.) toward increased risk of SSI among Black and low income patients. Overall, the prevalence of potential complications of SSIs was lower than for BSIs and pneumonia (Table 4), although these patients were almost 4 times more likely to be mechanically ventilated than non-infected patients $(21.2 \%$ vs. $5.4 \%, \mathrm{p}<.0001)$. In-hospital mortality was $21 \%$ more likely among these patients $(7.9 \%$ vs. $6.5 \%, \mathrm{p}$ $=$ n.s.).

\section{Urinary Tract Infections}

UTI, the most common infection complicating STEMI, occurred in women more frequently than men (OR 2.46, 95\% CI 1.99-3.03, p <.0001) (Table 3). Each 5-year increase in patient age increased its risk by $27 \%$ (OR 1.27, 95\% CI 1.21-1.33, $\mathrm{p}<.0001)$. Co-morbidities and procedures associated with a higher prevalence of UTIs were: chronic kidney disease (OR 1.64, 95\% CI 1.26$2.14, \mathrm{p}<.01$ ), diabetes (OR 1.59, 95\% CI 1.30-1.96, $\mathrm{p}<$ .0001 ), COPD (OR 1.57, 95\% CI 1.24-2.00, $\mathrm{p}<.01$ ), dialysis (OR 2.24, 95\% CI 1.19-4.19, $\mathrm{p}<.05$ ), blood transfusion (OR 1.94, 95\% CI 1.43-2.64, p <.0001), cardiac surgical procedures (OR 1.83, 95\% CI 1.28-2.62, p < .01 ), and an indwelling intravascular catheter (OR 1.69, 95\% CI 1.17-2.43, $\mathrm{p}<.01$ ). UTI patients had a much higher prevalence of acute renal failure $(28.8 \%$ vs. $5.3 \%$, $\mathrm{p}<.0001)$ and heart failure $(50.6 \%$ vs. $18.0 \%, \mathrm{p}<.0001)$, and had a $69 \%$ higher risk of in-hospital death $(11.0 \%$ vs. $6.5 \%, \mathrm{p}<.0001)$ (Table 4).

\section{Discussion}

Our study revealed that $16.6 \%$ of STEMI patients hospitalized in Florida experienced HAI, approaching the $20.6 \%$ prevalence for ICU-acquired infections reported by Vincent et al. [13] and over three times the $5 \%$ rate reported in studies of AMI [14] and CCU patients $[7,15]$. Consistent with ICU patients with nosocomial infections [13,16], infected STEMI patients experienced prolonged hospital stays and were more likely to die prior to discharge. After multivariable adjustment, important factors associated with infection included older age, female gender, Black race, alcohol or drug use, and several serious co-morbidities. Older age, female gender, diabetes, and chronic kidney diseases were all strongly associated with the risk of UTI, consistent with an increased prevalence of asymptomatic bacteriuria and UTIs reported in elderly women with those comorbidities [17]. Urinary tract catheterization can potentiate the risk for serious and potentially life threatening UTIs in these patients.

Cigarette smoking was associated with a lower risk of HAI, despite well-known deleterious actions of cigarette smoke [18]. A possible explanation is that AMI patients who smoke tend to be younger than non-smokers and may possess fewer comorbidities, reducing their likelihood of infection [19]. This observed lower risk of infection in smokers may contribute to the reduced mortality rates reported by us [20] and others [21], especially since the majority of STEMI patients who smoked were younger than those who did not smoke.

Major procedures, especially dialysis, cardiac surgical procedures, blood transfusions, and indwelling arterial or venous catheters were associated with a higher prevalence of infection. While insertion, catheter number, and implantation duration of indwelling catheters increase the risk of both insertion site and systemic infections, catheter use is also a marker of the severity of the patient's underlying condition.

Significantly, PCI was found to be associated with a much lower risk of HAI, particularly SSI. In contrast, cardiac catheterization was associated with an increased risk of SSI. While the risk of infection due to both cardiac catheterization and PCI is low $(<1 \%)$, a greater risk occurs with PCI, especially when complicated $[22,23]$. This paradoxical effect may be explained by cardiac catheterization being a surrogate marker for other HAIincreasing procedures, e.g. cardiac surgical procedures, or the complications related to non-reperfusion therapy. Only $4.2 \%$ of the total cohort received thrombolysis. Demographic and comorbid factors could confound the association of catheterization with HAI, e.g. approximately $64 \%$ of those not receiving catheterization were $\geq 65$ years old compared to $42 \%$ of those who did, and all analyzed co-morbidities occurred slightly more 
Table 3 Multivariable adjusted odds ratios for predictors of infection among STEMI Patients $(n=11,879)^{*},{ }^{\dagger}$ at acute care hospitals in Florida, 2006.

\begin{tabular}{|c|c|c|c|c|c|}
\hline & $\begin{array}{c}\text { All Infections } \\
\mathrm{n}=1,969 \\
\text { Odds-Ratio (95\% } \\
\mathrm{Cl})\end{array}$ & $\begin{array}{c}\text { Blood Stream } \\
\text { Infection }^{3} \\
n=310 \\
\text { Odds Ratio }(95 \% \mathrm{Cl})\end{array}$ & $\begin{array}{c}\text { Pneumonia }^{\ddagger} \\
n=550 \\
\text { Odds Ratio (95\% } \\
\text { Cl) }\end{array}$ & $\begin{array}{c}\text { Surgical Site } \\
\text { Infection }^{\neq} \\
\mathrm{n}=491 \\
\text { Odds Ratio (95\% } \\
\text { CI) }\end{array}$ & $\begin{array}{c}\text { Urinary Tract } \\
\text { Infection }^{\neq} \\
\mathrm{n}=708 \\
\text { Odds Ratio (95\% } \\
\text { Cl) }\end{array}$ \\
\hline \multicolumn{6}{|l|}{ Patient Characteristics } \\
\hline \multicolumn{6}{|l|}{ Age } \\
\hline Each 5 year increase & $\begin{array}{c}1.11(1.00-1.14) \\
\quad \neq \neq\end{array}$ & $1.06(0.95-1.17)$ & $1.21(0.14-1.28)$ & $0.99(0.94-1.04)$ & $1.27(1.21-1.33)^{* *}$ \\
\hline \multicolumn{6}{|l|}{ Sex } \\
\hline Female & $1.29(1.15-1.44)^{* *}$ & $0.71(0.43-1.21)$ & $0.83(0.64-1.06)$ & $0.80(0.62-1.04)$ & $2.46(1.99-3.03)^{* *}$ \\
\hline \multicolumn{6}{|l|}{ Race/Ethnicity } \\
\hline Black race & $\begin{array}{c}1.23(1.00-1.51) \\
\neq \neq\end{array}$ & $1.27(0.54-2.97)$ & $1.19(0.75-1.90)$ & $1.44(0.98-2.11)$ & $1.01(0.67-1.52)$ \\
\hline Hispanic ethnicity & $1.05(0.88-1.25)$ & $1.92(0.99-3.73)$ & $0.98(0.65-1.47)$ & $0.84(0.57-1.22)$ & $1.02(0.70-1.42)$ \\
\hline \multicolumn{6}{|l|}{ Income by Proxy } \\
\hline $\begin{array}{l}\text { Low income (Medicaid, self-pay, } \\
\text { uninsured) }\end{array}$ & $1.14(0.97-1.34)$ & $1.36(0.69-2.71)$ & $1.07(0.73-1.58)$ & $1.30(0.97-1.73)$ & $1.13(0.78-1.64)$ \\
\hline \multicolumn{6}{|l|}{ Patient Risk Factors } \\
\hline Cigarette smoking & $\begin{array}{c}0.76(0.66-0.87) \\
+\dagger\end{array}$ & $0.62(0.32-1.22)$ & $1.16(0.86-1.56)$ & $0.70(0.53-0.91)+\dagger$ & $0.66(0.48-0.82) \neq \neq$ \\
\hline Alcohol abuse & $\begin{array}{c}1.73(1.30-2.31) \\
+†\end{array}$ & $0.94(0.20-4.44)$ & $2.31(1.33-.01)+\dagger$ & $1.67(0.96-2.90)$ & $0.93(0.37-2.36)$ \\
\hline Drug abuse & $\begin{array}{c}1.55(1.09-2.20) \\
\quad \neq \neq\end{array}$ & $1.80(0.37-8.80)$ & $0.64(0.22-1.84)$ & $1.02(0.52-2.00)$ & $1.38(0.48-3.84)$ \\
\hline \multicolumn{6}{|l|}{ Patient Co-Morbidities } \\
\hline Chronic bronchitis & $2.39(1.84-3.09)^{* *}$ & $2.56(0.87-7.52)$ & $4.30(2.82-.56)^{* *}$ & $1.17(0.54-2.53)$ & $1.47(0.85-2.53)$ \\
\hline $\begin{array}{l}\text { Chronic obstructive pulmonary disease } \\
\text { (COPD) }\end{array}$ & $2.03(1.79-2.30)^{* *}$ & $1.80(1.03-3.14)$ 㧊 & $2.28(1.75-.96)^{* *}$ & $1.22(0.90-1.65)$ & $1.57(1.24-2.00) \mathrm{t \dagger}$ \\
\hline Diabetes & $1.33(1.19-1.49)^{* *}$ & $0.94(0.56-1.58)$ & $1.10(0.85-1.42)$ & $1.24(0.98-1.58)$ & $1.59(1.30-1.96)^{* *}$ \\
\hline Chronic Kidney Diseases $^{\S}$ & $1.47(1.26-1.72)^{* *}$ & $1.72(0.92-3.23)$ & $1.43(1.04-.98)$ 㧊 & $0.85(0.54-1.32)$ & $1.64(1.26-2.14)+\dagger$ \\
\hline Cardiomyopathy & $1.50(1.25-1.80)^{* *}$ & $1.55(0.75-3.22)$ & $1.20(0.80-1.81)$ & $1.24(0.81-1.91)$ & $1.26(0.89-1.71)$ \\
\hline Valve disorders & $\begin{array}{c}1.17(1.01-1.37) \\
\quad \neq \neq\end{array}$ & $2.22(1.26-3.92)+\dagger$ & $1.22(0.88-1.68)$ & $0.94(0.64-1.39)$ & $0.91(0.69-1.22)$ \\
\hline \multicolumn{6}{|l|}{ Patient Procedures } \\
\hline Cardiac catheterization & $1.10(0.93-1.30)$ & $0.51(0.25-1.04)$ & $0.79(0.55-1.15)$ & $2.89(1.98-4.22)^{* *}$ & $1.07(0.77-1.47)$ \\
\hline $\begin{array}{l}\text { Percutaneous coronary intervention } \\
\text { (PCl) }\end{array}$ & $\begin{array}{c}0.81(0.69-0.95) \\
+†\end{array}$ & $1.05(0.53-2.08)$ & $0.85(0.59-1.21)$ & $0.63(0.46-0.88)+\dagger$ & $0.85(0.63-1.16)$ \\
\hline Cardiac surgical procedures & $1.53(1.28-1.84)^{* *}$ & $1.42(0.65-3.14)$ & $1.54(1.02-.33) \#$ & $0.66(0.43-1.01)$ & $1.83(1.28-2.62)$ t十 \\
\hline Indwelling arterial or venous catheter & $3.90(3.29-4.63)^{* *}$ & $6.99(3.93-12.41)^{* *}$ & $3.93(2.79-.55)^{* *}$ & $1.98(1.30-3.03)+\dagger$ & $1.69(1.17-2.43)+\dagger$ \\
\hline Pacemaker I & $1.02(0.74-1.40)$ & $0.63(0.14-2.80)$ & $1.41(0.76-2.58)$ & $1.43(0.79-2.57)$ & $0.61(0.30-1.23)$ \\
\hline Dialysis & $3.40(2.40-4.82)^{* *}$ & $6.97(2.81-17.26)^{* *}$ & $3.55(1.76-.09)+\dagger$ & $2.61(1.10-.17) \neq \neq$ & $2.24(1.19-4.19)$ 㧊 \\
\hline Blood Transfusion & $1.64(1.37-1.96)^{* *}$ & $2.10(1.07-4.11)$ 㧊 & $0.90(0.58-1.40)$ & $1.62(1.08-.45) \neq \neq$ & $1.94(1.43-2.64)^{* *}$ \\
\hline
\end{tabular}

*Excludes patients who were transferred to another hospital.

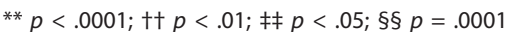

†All models compared to non-infected STEMI patients and are adjusted for all other variables in the table.

¥Patients can appear in more than one infection category.

§Includes hypertensive chronic kidney disease, end-stage renal disease (ESRD), unspecified chronic kidney disease and unspecified chronic renal failure.

| |lncludes temporary/permanent pacemakers and implantable defibrillators.

frequently $(60.2 \%$ vs. $39.8 \%)$ in non-catheterized patients. These differences were addressed in the regression analysis. Thus, the lower risk associated with PCI could reflect the over-riding benefit of this STEMI treatment. PCI patients suffer less myocardial damage, resulting in improved hemodynamic and clinical states, leading to shorter ICU/CCU stays and earlier hospital discharge, contributing to reduced HAI exposure. 
Table 4 Potential complications of infection among hospitalized STEMI patients $(n=11,879)$ * in Florida, 2006.

\begin{tabular}{|c|c|c|c|c|c|c|}
\hline & $\begin{array}{c}\text { Non-Infected STEMI } \\
\text { Patients } \\
n=9,910 \\
\%(n)\end{array}$ & $\begin{array}{c}\text { Blood Stream } \\
\text { Infection } \\
\mathrm{n}=310^{+} \\
\%(n)\end{array}$ & $\begin{array}{c}\text { Pneumonia } \\
n=550 \\
\%(n)\end{array}$ & $\begin{array}{c}\text { Surgical Site } \\
\text { Infection } \\
\mathrm{n}=491 \\
\%(n)\end{array}$ & $\begin{array}{l}\text { Urinary Tract } \\
\text { Infection } \\
\mathrm{n}=708 \\
\%(n)\end{array}$ & $\begin{array}{c}\text { All Other } \\
\text { Infections } \\
\mathrm{n}=503^{*} \\
\%(n)\end{array}$ \\
\hline \multicolumn{7}{|c|}{$\begin{array}{l}\text { Potential Complications of } \\
\text { Infection }^{\S}\end{array}$} \\
\hline Acute renal failure & $5.3(526)$ & $51.3(158)^{* *}$ & $30.6(168)^{* *}$ & $15.5(76)^{* *}$ & $28.8(204)^{* *}$ & $23.1(116)^{* *}$ \\
\hline Heart failure & $18.0(1776)$ & $58.8(181)^{* *}$ & $63.5(349)^{* *}$ & $26.9(132)^{* *}$ & $50.6(358)^{* *}$ & $46.9(236)^{* *}$ \\
\hline Swan-Ganz catheter & $1.3(129)$ & $8.8(27)^{* *}$ & $5.1(28)^{* *}$ & $3.9(19)^{* *}$ & $4.0(28)^{* *}$ & $5.0(25)^{* *}$ \\
\hline Mechanical ventilation & $5.4(537)$ & $14.9(46)^{* *}$ & $13.1(72)^{* *}$ & $21.2(104)^{* *}$ & $8.5(60)^{* *}$ & $25.1(126)^{* *}$ \\
\hline Intensive care unit stay & $64.0(6339)$ & $69.8(215)$ 㧊 & $\begin{array}{c}69.3(381) \\
\quad \neq \neq\end{array}$ & $68.2(335)$ 㧊 & $70.6(500) \wedge$ & $67.8(341)$ \\
\hline Length of stay $7+$ days & $15.5(1535)$ & $75.7(233)^{* *}$ & $65.5(360)^{* *}$ & $44.8(220)^{* *}$ & $57.6(408)^{* *}$ & $61.6(310)^{* *}$ \\
\hline In-hospital mortality & $6.5(640)$ & $31.3(97)^{* *}$ & $19.6(108)^{* *}$ & $7.9(39)$ & $11.0(78)^{* *}$ & $7.4(37)$ \\
\hline
\end{tabular}

* Excludes patients who were transferred to another hospital.

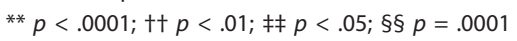

$\dagger$ Patients can appear in more than one infection category.

‡ Includes lower respiratory, gastrointestinal, cardiovascular, bone and joint, ear, eye, nose \& throat, skin and soft tissue, and site unspecified bacterial infections.

$\S$ Chi-square tests of homogeneity were conducted to compare potential complicating factors of each infection to non-infected STEMI patients.

\section{Study Limitations}

Limitations of our study include the potential for misclassification of both secondary diagnosis and procedures codes. Sherman et al. reported that administrative data reviews were not as accurate as targeted active surveillance at identifying HAIs [11]. Major procedures performed in surgical wards are likely to be correctly ICD9-CM coded; however, minor procedures performed in patients' rooms or in radiology departments are often inaccurately coded or missing [24]. Consequently, we could not evaluate the prevalence of indwelling urinary catheters, a minor procedure rarely reported. In our 2006 dataset, "present on admission" indicator variables were incomplete and could not be analyzed. These coding inaccuracies may persist, because as of October 2007 Medicare no longer reimburses hospitals for treatment costs incurred by HAIs [25]. Thus, the prevalence of HAIs is likely underestimated.

Our study could not definitively establish a temporal association among co-morbidities, procedures, and development of infections. This limitation should be addressed in future studies with more detailed clinical data, preferably by medical chart review. However, even with chart review, temporality may often be difficult to establish, because the detailed physical exams and multiple tissue cultures necessary to establish "present on admission" infections may not routinely be completed for STEMI patients in many hospitals. This limitation particularly applies to UTIs, which are not rare in community-dwelling populations and are often asymptomatic in elderly women [17], but is less problematic than for other types of infections. Finally, any selection biases of patients for PCI and the relationship of the timing of PCI to admission could not be determined.

\section{Conclusions}

We conclude that: 1) Approximately one sixth of STEMI patients hospitalized in Florida develop an HAI, a prevalence rate approaching that for ICU patients, often resulting in prolonged hospital stays and adverse clinical outcomes including in-hospital mortality; 2) BSIs and pneumonia were associated with the greatest likelihood of prolongation of hospital stay and in-hospital mortality; and 3) PCI was associated with reduced risk of overall infection, especially SSI. While current STEMI treatment guidelines do not emphasize risk of HAI $[26,27]$, improved surveillance, prevention, and treatment for high risk patients may reduce the risk. Despite limitations inherent in this type of study, the lower risk of infection associated with PCI provides additional support for the use of primary PCI for STEMI therapy.

\section{Acknowledgements and Funding}

An earlier version of this study was presented at the American Heart Association's (AHA) Scientific Sessions 2009 in Orlando, Florida. It was funded in part by a Grant-in-Aid to Dr. Pathak from the AHA.

\section{Author details}

'Department of Epidemiology and Biostatistics, College of Public Health, University of South Florida, (13201 Bruce B. Downs Blvd.), Tampa, (33612), USA. ${ }^{2}$ Department of Internal Medicine, College of Medicine, University of South Florida, (12901 Bruce B. Downs Blvd.), Tampa, (33612), USA.

\section{Authors' contributions}

EB and MN completed the data analysis, but each author contributed equally to the research design and implementation. All authors have read and approved the final manuscript.

\section{Competing interests}

The authors declare that they have no competing interests.

Received: 24 May 2011 Accepted: 22 November 2011 Published: 22 November 2011 


\section{References}

1. Ober S, Craven G: Will reporting healthcare-associated infection data change practice? The role for infusion nurses. J Infus Nurs 2008, 31(4):209-210.

2. Burke JP: Infection control - a problem for patient safety. N Engl J Med 2003, 348(7):651-656.

3. Board PHCC: Hospital acquired infections in Pennsylvania: Data reporting period 2006-2007.1-53.

4. Graves N, Tong E, Morton AP, Halton K, Curtis M, Lairson D, Whitby M: Factors associated with health care-acquired urinary tract infection. Am J Infect Control 2007, 35(6):387-392.

5. Rojo D, Pinedo A, Clavijo E, Garcia-Rodriguez A, Garcia V: Analysis of risk factors associated with nosocomial bacteraemias. J Hosp Infect 1999 42(2):135-141.

6. Lloyd-Jones D, Adams R, Carnethon M, De Simone G, Ferguson TB, Flegal K, Ford E, Furie K, Go A, Greenlund K, et al: Heart disease and stroke statistics-2009 update: a report from the American Heart Association Statistics Committee and Stroke Statistics Subcommittee. Circulation 2009, 119(3):e21-181.

7. Richards MJ, Edwards JR, Culver DH, Gaynes RP: Nosocomial infections in coronary care units in the United States. National Nosocomial Infections Surveillance System. Am J Cardiol 1998, 82(6):789-793.

8. Lepelletier D, Perron S, Bizouarn P, Caillon J, Drugeon H, Michaud JL, Duveau D: Surgical-site infection after cardiac surgery: incidence, microbiology, and risk factors. Infect Control Hosp Epidemiol 2005, 26(5):466-472.

9. Hollenbeak CS, Murphy DM, Koenig S, Woodward RS, Dunagan WC, Fraser VJ: The clinical and economic impact of deep chest surgical site infections following coronary artery bypass graft surgery. Chest 2000, 118(2):397-402.

10. Horan TC, Andrus M, Dudeck MA: CDC/NHSN surveillance definition of health care-associated infection and criteria for specific types of infections in the acute care setting. Am J Infect Control 2008, 36(5):309-332.

11. Sherman ER, Heydon KH, St John KH, Teszner E, Rettig SL, Alexander SK, Zaoutis TZ, Coffin SE: Administrative data fail to accurately identify cases of healthcare-associated infection. Infect Control Hosp Epidemiol 2006, 27(4):332-337.

12. Lynch JP: Hospital-acquired pneumonia: risk factors, microbiology, and treatment. Chest 2001, 119(2 Suppl):373S-384S.

13. Vincent JL, Bihari DJ, Suter PM, Bruining HA, White J, Nicolas-Chanoin MH, Wolff M, Spencer RC, Hemmer M: The prevalence of nosocomial infection in intensive care units in Europe. Results of the European Prevalence of Infection in Intensive Care (EPIC) Study. EPIC International Advisory Committee. JAMA 1995, 274(8):639-644.

14. Grandini LC Jr, Caramelli B: Infection complication portends poor prognosis in acute myocardial infarction. Arq Bras Cardiol 2006, 87(3):267-274.

15. Schandorf WA, Brown RB, Sands M, Hosmer D: Infections in a coronary care unit. Am J Cardiol 1985, 56(12):757-759.

16. Olaechea PM, Ulibarrena MA, Alvarez-Lerma F, Insausti J, Palomar M, De la Cal MA: Factors related to hospital stay among patients with nosocomial infection acquired in the intensive care unit. Infect Control Hosp Epidemiol 2003, 24(3):207-213.

17. Foxman B: Epidemiology of urinary tract infections: incidence, morbidity, and economic costs. Am J Med 2002, 113(Suppl 1A):5S-13S.

18. Arcavi $\mathrm{L}$, Benowitz NL: Cigarette smoking and infection. Arch Intern Med 2004, 164(20):2206-2216.

19. Gourlay SG, Rundle AC, Barron HV: Smoking and mortality following acute myocardial infarction: results from the National Registry of Myocardial Infarction 2 (NRMI 2). Nicotine Tob Res 2002, 4(1):101-107.

20. Pathak EB, Strom JA: Percutaneous coronary intervention, comorbidities, and mortality among emergency department-admitted ST-elevation myocardial infarction patients in Florida. J Interv Cardiol 2010, 23(3):205-215.

21. Katayama T, Iwasaki Y, Sakoda N, Yoshioka M: The etiology of 'smoker's paradox' in acute myocardial infarction with special emphasis on the association with inflammation. Int Heart J 2008, 49(1):13-24.

22. Baim D, (ed.): Grossman's Cardiac Catheterization, Angiography, and Intervention. New York: Lippincott/Williams \& Wilkins; 72005.
23. Munoz P, Blanco JR, Rodriguez-Creixems M, Garcia E, Delcan JL, Bouza E: Bloodstream infections after invasive nonsurgical cardiologic procedures. Arch Intern Med 2001, 161(17):2110-2115.

24. Quan H, Parsons GA, Ghali WA: Validity of procedure codes in International Classification of Diseases, 9th revision, clinical modification administrative data. Med Care 2004, 42(8):801-809.

25. Preventable hospital-acquired conditions (HACs), including infections. Edited by: 48471 FR 2008.

26. Antman EM, Anbe DT, Armstrong PW, Bates ER, Green LA, Hand M, Hochman JS, Krumholz HM, Kushner FG, Lamas GA, et al: ACC/AHA guidelines for the management of patients with ST-elevation myocardial infarction: a report of the American College of Cardiology/American Heart Association Task Force on Practice Guidelines (Committee to Revise the 1999 Guidelines for the Management of Patients with Acute Myocardial Infarction). Circulation 2004, 110(9):e82-292.

27. Antman EM, Hand M, Armstrong PW, Bates ER, Green LA, Halasyamani LK, Hochman JS, Krumholz HM, Lamas GA, Mullany CJ, et al: 2007 focused update of the ACC/AHA 2004 guidelines for the management of patients with ST-elevation myocardial infarction: a report of the American College of Cardiology/American Heart Association Task Force on Practice Guidelines. J Am Coll Cardiol 2008, 51(2):210-247.

\section{Pre-publication history}

The pre-publication history for this paper can be accessed here: http://www.biomedcentral.com/1471-2261/11/69/prepub

doi:10.1186/1471-2261-11-69

Cite this article as: Nash et al:: Prevalence of major infections and adverse outcomes among hospitalized. ST-elevation myocardial infarction patients in Florida, 2006. BMC Cardiovascular Disorders 2011 11:69.

\section{Submit your next manuscript to BioMed Central and take full advantage of:}

- Convenient online submission

- Thorough peer review

- No space constraints or color figure charges

- Immediate publication on acceptance

- Inclusion in PubMed, CAS, Scopus and Google Scholar

- Research which is freely available for redistribution

Submit your manuscript at www.biomedcentral.com/submit
C) Biomed Central 